Donato Bergandi

\title{
Environnement, éthique et politique : les limites d'une démocratie inaboutie et leurs conséquences néfastes sur la protection de la nature
}

\begin{abstract}
Avertissement
Le contenu de ce site relève de la législation française sur la propriété intellectuelle et est la propriété exclusive de l'éditeur.

Les œuvres figurant sur ce site peuvent être consultées et reproduites sur un support papier ou numérique sous réserve qu'elles soient strictement réservées à un usage soit personnel, soit scientifique ou pédagogique excluant toute exploitation commerciale. La reproduction devra obligatoirement mentionner l'éditeur, le nom de la revue, l'auteur et la référence du document.

Toute autre reproduction est interdite sauf accord préalable de l'éditeur, en dehors des cas prévus par la législation en vigueur en France.
\end{abstract}

revues.org

Revues.org est un portail de revues en sciences humaines et sociales développé par le Cléo, Centre pour l'édition électronique ouverte (CNRS, EHESS, UP, UAPV).

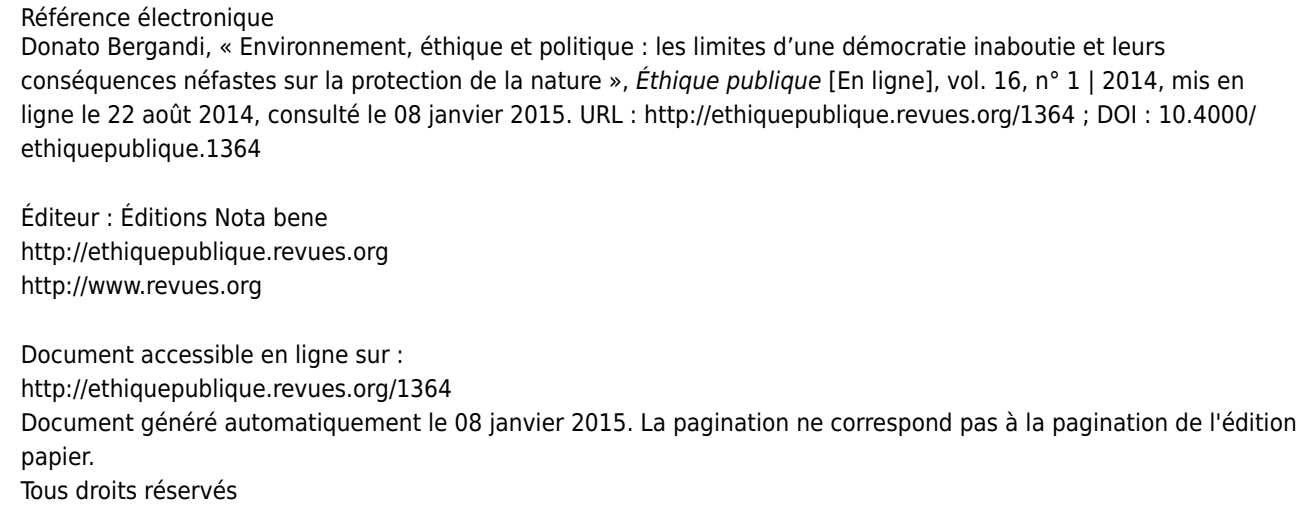




\section{Donato Bergandi}

\section{Environnement, éthique et politique : les limites d'une démocratie inaboutie et leurs conséquences néfastes sur la protection de la nature}

Caste : Groupe qui se distingue par ses privilèges et son esprit d'exclusive à l'égard de toute personne qui n'appartient pas au groupe.

Larousse

Au cours du temps, des élites religieuses, aristocratiques, militaires ou politico-économiques se sont succédé dans la gestion de nos sociétés parfois au nom d'un dieu, parfois au nom d'un idéal supérieur, et abstrait, par exemple, celui de la nation et parfois, plus récemment, au nom de la souveraineté populaire ou de la démocratie.

Les conditions de vie ont changé avec le temps et le mépris des monarques, des princes et des prêtres pour la populace, cette masse informe, inculte et brute a disparu, mais il serait plus juste de dire que l'ancien mépris, avec l'avènement de la démocratie représentative moderne, a pris d'autres formes plus adaptées au monde actuel. Il s'est transmuté comme par magie en un respect publiquement affiché, et parfois grossièrement, du suffrage universel et de la souveraineté populaire de la part des politiques professionnels. Toutefois la relation de subordination entre le peuple et les élites n'a pas disparu pour autant, elle s'est plutôt transformée en une délégation de pouvoir consciemment consentie.

La démocratie représentative n'est pas synonyme de démocratie. Elle représente seulement l'une des expressions possibles du régime politique démocratique. D'autres modèles de démocratie tels que la démocratie directe ou la démocratie participative (ou délibérative) font partie des options possibles de développement politique de nos sociétés.

La démocratie, dès ses origines athéniennes, s'est présentée comme une révolution politique et culturelle capable d'instaurer une nouvelle manière de penser, de parler et d'agir. Entre 508 et 322 avant notre ère, sur la base de la révolte du peuple athénien contre les oligarques soutenus par les Spartiates, la démocratie s'est installée à Athènes, c'est-à-dire, un « pouvoir politique activement et collectivement exercé par le peuple »(Ober, 1996, 19 sqq.). La démocratie athénienne était une démocratie directe. Elle représente le modèle pour un régime politique où l'ensemble des citoyens participe directement, c'est-à-dire sans médiation d'une élite politique, à la définition des lois de l'état.

La démocratie participative, souvent présentée comme une forme de renouvellement de la démocratie représentative, se trouve dans un espace intermédiaire entre la démocratie directe et la démocratie représentative. Le modèle de la démocratie participative est fortement influencé par la théorie de l'agir communicationnel de Jürgen Habermas (1981) qui lie dans un seul cadre interprétatif les dynamiques des processus culturels, sociaux et psychologiques et qui représente la base de sa théorie de la société. L'action communicationnelle, expression d'un contexte social coopératif, possède des fonctions sociétales telles que la compréhension réciproque, l'intégration sociale et la formation de la personnalité individuelle (1981, vol. 2 : 88) et permet de parvenir à un « consensus rationnellement fondé » dans les domaines cognitif, éthique et politique (1981, vol. 2 : 107, 126-127). Au centre de la théorie des interactions linguistiques et sociales de Habermas se retrouve l'idée que les acteurs sociaux confrontés à une situation problématique réussiront à parvenir à un consensus « pourvu que » (as long as) leur attitude communicationnelle ne soit pas imprégnée d'une orientation tournée vers le succès individuel, mais qu'elle ait comme but la compréhension réciproque (1981, vol. 2 : 262).

C'est sur cette base que Habermas (1996) structurera son schéma communicationnel d'une société fondée sur des procédures politiques délibératives permettant la formation d'une opinion publique et d'une volonté politique rationnelles, chez les individus et dans la 
collectivité. Habermas retrouve, à la base des procédures démocratiques, le principe qu'aux intérêts de chaque personne soit donné une considération égale (1996:109) et le principe selon lequel la légitimité démocratique d'un statut législatif résulte de « l'assentiment » de l'ensemble des citoyens dans le cadre d'un processus législatif basé sur la délibération (1966: 110). Au cœur de ce modèle de démocratie participative, il existe ce que l'on peut nommer une «tension communicationnelle » entre le centre (les instances représentatives élues par les citoyens) et la périphérie (la société civile). Habermas structure son modèle de politique délibérative autour de l'influence que la « sphère publique » peut exercer sur les instances politiques qui prendront les décisions politiques finales. La sphère publique est constituée par l'ensemble des acteurs de la société civile qui grâce à différentes formes de communication et de discussion dans l'agora publique, cherchent à faire valoir leurs intérêts, valeurs ou options politiques et donc à avoir un « effet rétroactif influent »sur les instances politiques décisionnelles. La dynamique communicationnelle entre les représentants des citoyens et la société civile devrait être en mesure d'engendrer une «syntonie politique » capable de produire des décisions rationnelles et fonctionnelles pour le bien commun (1996 : 350-358, 380-383, $442)^{1}$.

Mais pourquoi parler de la structure et des procédures de la gouvernance démocratique lorsqu'on analyse les politiques publiques d'aménagement du territoire et de protection de la biodiversité à l'échelle planétaire? Parce que, d'une part, les élites politiques fondent leur légitimité sur le suffrage universel basé sur la participation des populations au vote et, d'autre part, selon les constitutions démocratiques, les représentants élus du peuple « sont tenus » de réaliser des politiques publiques qui tiennent compte de la volonté et des besoins des citoyens électeurs qui les ont élus. Et cela est d'autant plus vrai lorsqu'il s'agit de questions environnementales, c'est-à-dire de « biens communs » (air, eau, sol, processus de régulation écologique, gestion de la biodiversité...) qui, par définition, ne sont pas, ou ne devraient pas être, l'apanage d'individus ou de groupes économiques particuliers. est constitué des multiples facettes de la crise environnementale, il y a désormais l'obligation scientifique, morale et politique, de constater qu'aux échelons national et international, une tendance évolutive se manifeste de plus en plus comme l'un des signes d'une impasse majeure du système actuel de démocratie représentative : Les élites politiques forment avec les élites économiques une seule et unique classe politico-économique ou caste qui tend à suivre des logiques d'action et des finalités le plus souvent contraires à la volonté et aux intérêts de la population ainsi qu'au maintien des équilibres de la biosphère et à la protection de la biodiversité.

Il existe de très nombreux exemples qui illustrent la façon dont le système politique de la démocratie représentative et le système économique libéral se sont matérialisés dans une véritable collusion, ou entente tacite, entre les élites politiques et les élites économiques pour donner vie à une véritable caste oligarchique, nationale et internationale, absolument imperméable aux finalités de justice sociale, économique et environnementale présentes dans les constitutions démocratiques. Cette caste constituée d'une composante politique et d'une composante économique (entrepreneurs industriels, groupes économiques et financiers...) assume des positions substantiellement unitaires et tendanciellement autocratiques lorsqu'il s'agit de défendre ses privilèges et de gérer la res publica. Et tout cela se réalise tantôt à l'échelle nationale, tantôt à l'échelle internationale, et s'organise « indépendamment » des valeurs philosophico-politiques de référence affichées par les composantes politiques de ladite caste.

10 Il existe un lien direct entre la crise environnementale et la gestion de la res publica par de cette caste oligarchique qui privilégie des intérêts particuliers aux intérêts des populations. Cette prédilection à court terme des intérêts de la caste politico-économique ne tient compte que de manière marginale du droit d'exister des autres espèces non humaines et de la nécessaire persistance des environnements et des systèmes de régulation écologique, qui ont permis à la biodiversité, dans son ensemble et pendant des millions d'années, d'évoluer durablement sur la planète. Certains pourraient douter de l'existence d'une telle élite ou caste oligarchique 
politico-économique responsable des affaires économiques de la planète tenue, entre autres, d'affronter et, éventuellement, de résoudre la question environnementale.

À toutes fins utiles, rappelons que Karl Marx (1818-1883) avait clairement déterminé que le pouvoir politique résidait dans les mains d'une élite dont les intérêts étaient contraires aux intérêts de la population ([1871] 1975 : III ${ }^{2}$ ), sans oublier la critique extrêmement caustique du corporatisme des élites gouvernementales dans la gestion de la société qui a été faite par les initiateurs même du courant politique libéral. Jeremy Bentham (1748-1832), l'un des pères fondateurs avec John Stuart Mill (1806-1873) de l'utilitarisme philosophique moderne, considère « le principe d'utilité (ou de bien-être) » (greatest happiness principle) comme le fondement de sa conception de la morale et de la politique, un principe qui devrait présider à toute action ou décision individuelle ou gouvernementale, un principe qui devrait fonder la base de l'éthique publique de la nation. D'après Bentham, les décisions et les actions peuvent être jugées en fonction de leur conformité à l'axiome suivant : «[...] c'est le plus grand bonheur du plus grand nombre qui est la mesure du juste et de l'injuste » ([1776] 1996 : 86). Bentham soutient que chaque décision des gouvernants sera juste ou injuste selon les effets qu'elle aura sur l'ensemble de la communauté. La conformité au principe d'utilité des décisions gouvernementales se réalise quand la tendance à augmenter le bonheur de la communauté l'emporte sur les tendances qui pourraient le faire diminuer ([1789] $2011: 27)^{3}$. Dans le premier des trois tomes de Traités de législation civile et pénale, la signification du principe d'utilité et ses conséquences pour l'individu et pour la société dans son ensemble est clairement expliquée : «Ce qui est conforme à l'utilité ou à l'intérêt d'un individu, c'est ce qui tend à augmenter la somme totale de son bien-être. Ce qui est conforme à l'utilité ou à l'intérêt d'une Communauté, c'est ce qui tend à augmenter la somme totale du bien-être des individus qui la composent $\gg($ Bentham, $1802: 3$ ).

$\mathrm{Si}$ «l'utilité » ainsi définie représente la «mesure » du bien-être individuel et collectif, sa contrepartie négative est constituée par les sinister interests, des intérêts qui représentent la raison principale des déséquilibres moraux et politiques des individus et qui vont à l'encontre de l'intérêt collectif : « [...] on veut dire par "sinistre", [...] agissant ou tendant à agir dans une direction dévoyée, c'est-à-dire dans une direction telle qu'elle donne naissance à un acte mauvais, autrement dit, vicieux. » Ces sinister interests, ou «intérêts dévoyés » se concrétisent dans des actes «d'improbité » portant atteinte aux autres personnes (Bentham, [1815] 2008 : $\S \mathrm{V}, 36)$.

Mill considère que l'existence et la permanence de tout type de gouvernement, et en particulier le gouvernement représentatif démocratique, doivent satisfaire à trois conditions fondamentales. Le gouvernement doit 1) être voulu et accepté par le peuple, 2) doit, au besoin, être préservé et défendu par le peuple et 3) doit être activement soutenu par le respect des lois de la part de citoyens coopérant avec les autorités ([1861] 1877 : 8-14, 93-97). Idéalement, le but du gouvernement est d'agir politiquement de manière à renforcer l'exercice des facultés, des capacités intellectuelles, morales et d'action des citoyens, tout cela afin d'éviter l'un des dangers qui pèsent sur le gouvernement représentatif : « [...] le danger [d'être] sous l'influence d'intérêts non identiques au bien-être général de la communauté » ([1861] $1877: 144$ et 154-155). Mill, comme Bentham par ailleurs, est extrêmement lucide sur le fait qu'aucun type de gouvernement n'est immunisé contre les comportements des élites responsables de la gestion politique de la nation :

Donc, un des grands dangers de la démocratie comme de toutes les autres formes de gouvernement, consiste dans les intérêts sinistres de ceux qui possèdent le pouvoir : ce danger est celui d'une législation de classe, d'un gouvernement qui recherche (soit qu'il réussisse, soit qu'il échoue) le profit immédiat de la classe dominante, au détriment durable de la masse. Et c'est une chose à considérer entre toutes, lorsqu'on détermine la meilleure constitution d'un gouvernement représentatif, que la manière de se bien précautionner contre ce mal ([1861] 1877 : 167).

Pour contrer les menaces au summum bonum d'une gestion politique non conforme au principe d'utilité, Mill considère qu'une société bien fondée tant du point de vue de la morale individuelle que du point de vue de l'éthique publique sera en mesure d'harmoniser les intérêts individuels et collectifs en utilisant «l'éducation » et «l'opinion » de manière à ce que 
«l'homme ne concevrait même pas l'idée d'un bonheur personnel allié à une conduite pratique opposée au bien général » ([1863] $1889: 32)$.

Toujours dans le sillage de la pensée utilitariste et libérale, que dire alors du positionnement de Gifford Pinchot (1865-1946)? Forestier et gouverneur républicain (progressiste), Pinchot est à l'origine du mouvement de conservation des ressources à la base du paradigme du développement durable actuel (Bergandi et Galangau-Querat, 2008; Bergandi, 2009; Blandin, 2009; Bergandi et Blandin, 2012). Dans son texte de référence The Fight for Conservation, il a souligné à maintes reprises, les dangers de la collusion entre le monde de la politique et celui des affaires pour la conservation des ressources et leur développement $(1910: 26$, 79-80, 82-84). Il a aussi émis l'hypothèse - tristement confirmée depuis par l'histoire - que, si l'État n'est pas capable de s'y opposer, des monopoles seront en mesure de s'approprier les ressources naturelles et de pousser la classe politique à faire les lois servant leurs propres intérêts et non ceux de la population ou du « bien commun » (public good) (1910 : 24-30). Ou encore, plus tard, que dire de l'alerte de Dwight David Eisenhower (1890-1969) concernant l'influence grandissante d'une élite particulière, issue de la conjonction entre structures étatiques et lobbies des armements? Eisenhower, général et président des États-Unis (1953-1961), dans son discours d'adieu adressé à la nation, exprimait les dangers potentiels de l'émergence d'une élite politico-militaro-industrielle capable de drainer d'énormes ressources, d'intervenir sur nos moyens d'existence et de représenter « le risque potentiel d'une désastreuse ascension d'un pouvoir illégitime » (Eisenhower, 1961).

Par rapport à l'existence ou non d'une élite ou d'une caste oligarchique politico-économique, deux options interprétatives s'affrontent dans les domaines des sciences politiques. Robert A. Dahl (1968 : 273) a défié la thèse de l'existence des ruling elites en proposant un test très exigeant pour en vérifier ou en infirmer l'existence, à partir des critères suivants : l'élite en question est-elle reconnaissable comme un «groupe bien défini »? Dans le cadre de décisions politiques d'importance majeure, les préférences politiques des élites se trouventelles en opposition aux préférences d'autres groupes politiques? Les préférences des élites «prévalent-elles régulièrement »? D'autres considèrent que les élites non seulement existent, mais également que l'histoire de l'humanité est celle de leur remplacement continu, du déclin des anciennes élites à la montée de nouvelles (Pareto, $1968: 36$, chap. 3 et 4; Rothkopf, 2008) et que depuis l'émergence des sociétés industrielles, les élites sont plus nombreuses et «stratégiques »du fait même du développement des structures socio-économiques et politiques (Keller, 1963 : chap. 3).

Enfin, pour revenir un instant sur le test proposé par Dahl, il est intéressant de noter que, tout en étant sûrement trop demandant - car les élites ne peuvent se permettre de faire « régulièrement » prévaloir leurs intérêts que dans un régime despotique -, il permet de mieux identifier certains traits caractéristiques des élites supposées. La caste politico-économique internationale actuelle peut aisément être décrite comme un groupe bien défini ayant des potentialités élevées de contrôle et d'orientation des décisions politiques de nos sociétés et de l'avenir de la biodiversité et des équilibres planétaires.

L'entente tacite entre les élites politiques et économiques est par définition un phénomène élusif. Inutile donc de chercher un traité d'alliance entre les représentants du peuple et les représentants du pouvoir économique et financier. Au mieux, est-il possible de donner des exemples emblématiques révélant la partie émergée de l'iceberg. L'ancien président des États-Unis George W. Bush (républicain) a signé le projet de loi, écrit avec la collaboration des compagnies pétrolières, Energy Policy Act (8 août 2005). Cette loi soutenue par les républicains et les démocrates, outre le fait qu'elle subventionne les compagnies pétrolières avec des milliards de dollars des contribuables américains, établit l'exemption pour ces compagnies du respect de la Clean Water Act (qui prévoit entre autres la réduction de la pollution liée aux sites d'extraction), permet de couper des forêts pour produire de l'énergie, d'intégrer les déchets industriels toxiques dans le ciment utilisable pour construire les bâtiments, de faire des forages dans des zones protégées en Alaska et de développer le programme d'extraction des gaz et des huiles de schiste. Tout cela en refusant de mettre en place des politiques favorables aux énergies renouvelables ou qui visent à limiter le 
réchauffement climatique. Barack Obama, l'actuel président (démocrate), a signé le projet de loi Farmer Assurance Provision (26 mars 2013, renommé par les opposants politiques Monsanto Protection Act). Ce projet, qui a été en partie écrit directement par Monsanto, établit qu'il ne sera pas possible d'arrêter les tests ou les ventes des organismes génétiquement modifiés (OGM), même dans le cas où la consommation de ces produits aura des conséquences néfastes sur la santé de la population.

Enfin, deux exemples de politiques économiques, encore en phase de développement, permettront d'apporter d'autres éléments qui soutiennent l'hypothèse de l'existence d'une caste politico-économique internationale, ou bien ils pourront être utilisés comme des exemples montrant le contraire. Il s'agit de la construction de l'oléoducKeystone XL pipeline reliant le Canada et les États-Unis et de la négociation du Partenariat transatlantique sur le Commerce et l'Investissement (TTIP-Brussels, 13 février 2013 EUCO 39/13).

Le projet de la société pétrolière TransCanada prévoit la construction d'un oléoduc qui transportera les huiles extraites des sables bitumeux de l'Alberta jusqu'au golfe du Mexique. La décision présidentielle devra trancher sur la faisabilité ou non du projet en tenant compte des impacts environnementaux et sociaux (pollution des eaux, impacts sur la biodiversité, risques pour la santé publique, émissions de gaz à effet de serre, nombre d'emplois effectifs...) $)^{4}$.

Le TTIP est un accord que le parlement européen et le gouvernement américain sont en train de négocier dans l'opacité la plus totale : cet accord de libre-échange aurait comme finalité de faire tomber les barrières commerciales entre les États-Unis et l'Union européenne. Il prévoit, entre autres, l'ouverture des marchés publics européens aux entreprises étatsuniennes, la possibilité pour celles-ci de porter plainte contre des États ou des collectivités territoriales lorsqu'elles considèrent que leur activité est entravée par les lois ou les décisions des États de l'Union européenne, l'affaiblissement des normes européennes de protection des consommateurs (dosage des pesticides, non-étiquetage des OGM, utilisation des hormones dans l'élevage...), et l'acceptation de la fracturation hydraulique sur l'ensemble du territoire européen.

\section{Préserver la nature n'est pas conserver les ressources}

The average sportsman, God bless him, loudly proclaims that his interest is in perpetuating duck hunting for posterity in general, and for his kids in particular. James W. KIMBALL ${ }^{5}$, The Scientific Monthly, 1951

Cette phrase de James W. Kimball peut être considérée comme une préfiguration ante litteram de ce qui sera plus tard nommé le développement durable. Pinchot est mort en 1946, mais sa bataille pour la conservation des ressources avait déjà contribué à forger au moins deux générations d'adeptes de la conservation. La relation au monde qui émerge de cette phrase résume, de manière imagée, le cœur de l'arrière-plan philosophique de la conception pinchotienne de la conservation des ressources. Tout y est. La référence à une entité supérieure (qui, de manière subliminale, suggère que le monde est fait pour l'homme), la conception de la nature comme réserve de ressources et parc de récréation à la disposition de l'humanité, la relation prédatrice au monde (prédatrice certes, mais raisonnable, autrement dit wise use) et la référence aux générations futures. Il faut aménager les ressources pour permettre à la génération actuelle et aux générations futures de chasser et d'exploiter la nature encore et encore. Dernier aspect à mettre en relief : la référence aux enfants, et donc à un supposé système d'éducation, implique la transmission d'une culture de l'exploitation prétendument rationnelle de la nature permettant d'en tirer profit de manière de plus en plus efficace.

De nos jours, le paradigme, à la fois scientifique, éthique et politique, du développement durable est préconisé par différents acteurs sociaux (institutions gouvernementales, institutions onusiennes, organismes non gouvernementaux, lobbies industriels, associations citoyennes...) comme le modèle d'action pour former la base des nouvelles formes de gestion des rapports entre les humains et l'environnement. En réalité, ce modèle est le résultat d'une longue histoire qui trouve ses sources dans la confrontation éthique et politique du mouvement de conservation des ressources avec le mouvement de préservation de la nature qui s'est développé à partir de la deuxième moitié du XIX ${ }^{\mathrm{e}}$ siècle en Europe et aux États Unis (Bergandi et Blandin, 2012). 
Ce modèle, sur les plans de l'éthique et de l'économie politique, véhicule une perspective propre à l'utilitarisme philosophique classique et au libéralisme économique. Lorsqu'on transpose la perspective morale utilitariste dans les termes des relations de l'homme avec son environnement, il en ressort que la conception utilitariste classique, qui représente la structure philosophique portante du modèle du développement durable, oriente ce modèle selon une perspective à la fois anthropocentrique et « ressourciste». Anthropocentrique, car le seul bonheur, ou bien-être, pris en compte sera, au mieux, celui de l'ensemble des individus de l'espèce humaine, et « ressourciste », car il considère que la nature, dans son essence, n'est rien d'autre qu'une réserve de ressources, et de moyens, utiles aux fins et au bien-être des humains. Et c'est exactement cet anthropocentrisme des ressources qui est à la base de la crise environnementale actuelle. D'une part, jusqu'à nos jours, cette vision du monde, sur une planète où près d'un milliard d'humains souffrent de malnutrition ou meurent de faim, n'a pas réussi à réaliser dans la réalité politique son axiome fondamental (greatest happiness of the greatest number). D' autre part, la vision du monde utilitariste sous-jacente au modèle du développement durable implique que la nature, fondamentalement, n'a pas d'autre fonction que celle de satisfaire les besoins humains, qu'ils soient économiques, récréatifs, scientifiques ou culturels.

En effet, on peut reconnaître ou attribuer à la nature une valeur qui ne se limite pas à la valeur instrumentale ou utilitaire, c'est-à-dire qu'on peut lui reconnaître, ou lui attribuer, une valeur intrinsèque ou inhérente. La valeur intrinsèque d'une entité peut être déclinée de différentes manières. Il existe une acception non relationnelle de la valeur intrinsèque, au sens qu'une entité peut être considérée comme douée de valeur intrinsèque quand sa valeur dépend, essentiellement, de certaines propriétés ou qualités qui la caractérisent (Moore, 1922 : 260). L'acception non instrumentale de la valeur intrinsèque maintient qu'une entité sera considérée comme douée de valeur intrinsèque quand elle est une fin en soi et non un moyen en fonction d'une fin à atteindre (Kant [1785] 1904 : 65). Enfin, l'acception objectiviste de la valeur intrinsèque affirme qu'une entité est douée de valeur intrinsèque quand sa valeur est indépendante de l'évaluation ou de l'appréciation d'un observateur (Dewey, 1944 : 452; Bergandi 2013). Il est à noter qu'à plusieurs reprises, dans des textes internationaux visant à préserver la biodiversité des impacts des activités humaines, la valeur intrinsèque des entités naturelles a été mise en avant pour promouvoir des modes de vie alternatifs au consumérisme destructeur actuel (Convention sur la diversité biologique, 1992; Charte de la Terre, 2000; Initiative pour une éthique de la biosphère, 2010).

Finalement, le modèle oxymoronique du développement durable balance dangereusement entre Janus et Utopie. Il s'agit d'un bricolage idéologique capable de faire coexister la préservation avec la conservation, la valeur intrinsèque de la biodiversité avec sa valeur instrumentale. Sa dimension utopique s'ancre dans l'espoir que le modèle démocratique sera repris par l'ensemble des populations humaines et que le plus grand nombre pourra ainsi satisfaire ses besoins.

28 Cette utopie ne s'est pas encore réalisée et il ne semble pas qu'elle se réalisera de sitôt. Dans son dernier ouvrage et testament autobiographique, Pinchot rappelle une fois de plus la nécessité, pour l'existence de la démocratie, de la lutte contre les monopoles : «Il est de l'essence même de la démocratie que le plus grand avantage pour chacun de nous est le mieux atteint à travers la prospérité commune de tous. Le monopole est la négation de cette grande vérité. »(1947: 506. Je traduis). Pinchot configure son utopie sur la base du contrôle démocratique des forces économiques et de la poursuite du public good. Ses paroles nous permettent d'apprécier à quel point le monde idéalisé par le père philosophique du développement durable, non seulement n'est pas une réalité aujourd'hui, mais qu'il ne se réalisera pas non plus demain, à moins que des changements structurels profonds n'interviennent dans nos procédures démocratiques et dans nos modèles de référence éthique et économique :

Tout comme le féodalisme, avec sa tyrannie, s'est finalement rendu intolérable, la ploutocratie aussi, avec sa domination sur l'homme par l'intermédiaire de l'argent, avec ses privations pour la plupart des gens et son luxe réservé à quelques-uns, avec son avidité et son injustice, doit suivre le même chemin. 
Il est temps pour l'Amérique et le monde de dépasser un ordre social dans lequel le profit non réglementé est la force motrice. Il est temps de passer à un ordre social dans lequel l'égalité des chances ne sera plus un rêve et deviendra une réalité.

Je ne prétends pas prédire exactement l'ordre qui se réalisera ou par quelles étapes il sera atteint. Quand cela viendra, je veux qu'il se réalise grâce au développement et non par la révolution.

Quand cela viendra, j'espère et je crois que le nouvel ordre sera fondé sur la coopération plutôt que sur le monopole, sur le partage plutôt que sur l'expropriation, et que l'entraide remplacera la loi de la jungle. Quand cela viendra, j'espère et je crois que les grandes concentrations non réglementées de richesse, avec leur énorme pouvoir de nuisance, ne seront plus autorisées à exister (1947 : 509. Je traduis).

\section{Conclusions}

Une démocratie, dans son essence, est l'expression d'un équilibre entre diverses procédures de gestion de la res publica (législatives, juridiques, gouvernementales, administratives) qui doivent être pensées, d'abord, en vue du bien-être général, puis respectées et mises en œuvre. Les procédures actuelles ne nous protègent pas suffisamment contre des « interprétations » du mandat des électeurs contraires au bien-être des populations et à la durabilité des équilibres de la planète. Le modèle de la démocratie délibérative, fréquemment présenté comme la panacée censée résoudre la perte de représentativité des instances démocratiques, a essuyé des critiques majeures qui, pour être contrecarrées, nécessitent des réponses bien convaincantes et tangibles. La délibération n'a pas nécessairement d'effet sur les questions normatives (Austen-Smith, 1990 : 126, 144-145). La délibération peut prendre des formes «pathologiques » quand la communication de la part de groupes d'intérêts particuliers réussit à induire chez les électeurs des croyances et des valeurs contraires à leurs intérêts ou bien quand elle réussit à changer la «perception » que les politiques professionnels ont des prédilections et des choix politiques réels des électeurs (Stokes, 1998 : 128-132, 135-136). Le but des débats politiques est de faire accepter les objectifs poursuivis par les partis et non de faire émerger la véritable signification des politiques publiques (Becker, 1982 : 104, cité par Habermas, 1996, 294).

Il n'est pas excessif de considérer que, globalement, les systèmes politiques de démocratie représentative actuels, sous des formes variées et multiformes, tendent à être des pseudodémocraties plutôt que des démocraties véritables. Cela est dû au fait que nos démocraties courent le risque permanent d'être kidnappées par une caste oligarchique politico-économique nationale et internationale qui fait des sinister interests, de ces intérêts dévoyés, sa priorité politique. L'habileté politique et communicationnelle réside dans le fait de masquer ces intérêts par la mise en avant, formelle, des intérêts du plus grand nombre. Seule une démocratie réellement aboutie, qui prendrait en compte les intérêts à long terme des populations humaines et la persistance des équilibres des systèmes naturels qui les soutiennent, sera en mesure de faire face à la crise environnementale actuelle et de préserver concrètement et durablement les systèmes écologiques.

31 L'avènement d'une telle démocratie est lié à l'émergence d'une nouvelle éthique publique. Cette démocratie devra être en mesure de se démarquer du manque de représentativité des pseudo-démocraties actuelles en s'appuyant sur des procédures nouvelles propres à une démocratie directe structurée autour d'au moins quatre éléments principaux : 1) réduction de la base représentative de la démocratie exercée par une élite de politiques professionnels et élargissement de la participation directe des populations à la gestion de la res publica, autrement dit, réduction notable du nombre de députés et de sénateurs, accompagnée de l'utilisation prédominante de l'outil référendaire (référendum d'initiative populaire, abrogatif) et des pétitions de lois populaires; 2) obligation de la part des « citoyens décideurs » de respecter le mandat pour lequel ils ont été élus; 3) révocation du mandat de l'élu si les orientations du mandat ne sont pas respectées; 4) limitation du nombre des mandats qui peuvent être exercés dans une vie (deux mandats). Exercer un mandat parlementaire ne devrait pas en effet être considéré comme une « profession », mais comme un « service » que l'élu rend à la communauté. Par ailleurs, la permanence à vie dans un parlement de politiques professionnels correspond à la mise en place d'un système qui n'est plus véritablement démocratique, mais qui s'est métamorphosé plutôt en une véritable " partitocratie », un 
système qui, traditionnellement, fait des relations claniques et de la constitution d'un pouvoir souterrain, parallèle et masqué son trait le plus caractéristique. L'émergence d'une telle élite de professionnels de la politique est la négation même d'une démocratie véritable. D'autant plus que cette élite se transforme en une véritable caste d'intouchables lorsqu'elle rallie les intérêts d'élites économiques, tendanciellement tout à fait oublieuses et insouciantes des enjeux environnementaux et des intérêts des populations.

Compte tenu de la capacité de l'espèce humaine à détruire la biodiversité planétaire directement par l'exploitation des espèces et la fragmentation grandissante des milieux et indirectement par les effets de ses activités industrielles sur les systèmes de régulation écologique globaux -, l'abandon de l'éthique anthropocentrique ressourciste sous-jacente à nos politiques de gestion de la nature se révèle, désormais, de plus en plus, comme une obligation morale, économique et politique.

Obligation morale, car nos systèmes de valeurs orientent nos actions dans le monde. Si l'on veut construire une relation nouvelle et différente à l'environnement, il est nécessaire de choisir une option éthique nous permettant d'envisager que l'humanité n'est pas à part du reste de la nature, comme beaucoup de nos mythes et religions nous l'ont appris pendant des siècles, mais que « nous sommes la nature » ou, comme le dirait Élisée Reclus : «L'homme est la nature prenant conscience d'elle-même » (1905, vol. I : 1). Obligation économique, car sur le long terme, la démarche actuelle d'exploitation des ressources, selon des techniques qui ne sont pas pensées en fonction d'un impact minimal sur les écosystèmes mais seulement en fonction du degré de profit que l'on peut en retirer à court et à moyen terme, est néfaste non seulement pour la biosphère, mais également pour les systèmes socioéconomiques eux-mêmes. Obligation politique, enfin, car la résilience de ce qu'on peut désormais nommer les socioécosystèmes n'est plus assurée par un système de représentativité de la souveraineté populaire qui déconnecte de plus en plus le bien-être environnemental des populations et la préservation des équilibres des systèmes naturels, des décisions prises par des politiques professionnels et plutôt orientées vers la préservation des privilèges et des intérêts d'une minorité influente. En conclusion, la confluence, d'une part, d'un paradigme éthique anthropocentré et ressourciste, sous-jacent au modèle du développement durable, qui considère la biodiversité essentiellement comme un moyen au service des finalités de l'humanité, et, d'autre part, d'un modèle de représentativité politique qui ne permet pas aux populations de contrôler « directement », dans des temps rapides et de façon efficace l'action politique de leurs représentants, crée un mélange explosif pour l'intégrité de la biodiversité planétaire et le bienêtre des populations humaines.

Afin de garantir le bien-être économique et environnemental des populations et l'intégrité de la biodiversité, une double transition, éthique et politique, est désormais indispensable. Parmi les multiples options éthiques possibles, il y a la possibilité d'orienter nos sociétés selon les normes d'une éthique en syntonie avec ce qui, d'un point de vue socio-environnemental, doit être considéré comme le summum bonum : la préservation des équilibres permettant la coévolution résiliente des socio-écosystèmes. Une telle éthique sera fondée sur la reconnaissance de la continuité ontologique et évolutive entre les humains et le reste de la nature et centrée autour de normes qui reconnaissent dans l'impact minimal des actions humaines sur la nature le fondement éthique des conduites d'action à poursuivre individuellement et collectivement. L'autre volet de la transition concerne le modèle politique le plus conforme au respect de la volonté générale et à la sauvegarde de l'intégrité de la biodiversité. Seule l'émergence d'une démocratie aboutie et véritable, la démocratie directe, sera en mesure de faire face efficacement aux sinister interests d'une caste politico-économique ou minorité influente capable à la fois de menacer l'intérêt général et le bien commun des populations ainsi que la préservation des équilibres écologiques planétaires. La participation directe des populations à la gestion de la res publica socio-ecologica est très vraisemblablement l'unique sauvegarde contre l'exploitation irrationnelle de la nature de la part de groupes industriels et financiers qui trouvent un allié naturel dans une élite politique trop souvent oublieuse des intérêts et du bien-être des citoyens qui l'ont élue. 


\section{Bibliographie}

AUSTEN-SMITH, David (1990), «Information Transmission in Debate », American Journal of Political Science, vol. $34, \mathrm{n}^{\circ} 1, \mathrm{p} .124-152$.

BECKER, Werner, (1982), Die Freiheit, die wir meinen: Entscheidungfur die liberate Demokratie, München/Zürich, Piper.

BENTHAM, Jeremy ([1776] 1996), Fragment sur le gouvernement. Manuel de sophismes politiques, traduction de Jean-Pierre Cléro, Bruxelles/Paris, Bruylant/L.G.D.J.

BENTHAM, Jeremy ([1789] 2011), Introduction aux principes de morale et de législation, traduction du Centre Bentham, M. Bozzo-Rey, A. Brunon-Ernst, E. de Champs, J.-P. Cléro, C. Laval, M.-L. Leroy, G. Tusseau, Paris, Vrin.

BENTHAM, Jeremy (1802), Traités de législation civile et pénale, t. I, Paris, Bossange, Masson et Besson.

BENTHAM, Jeremy ([1815] 2008), Table des ressorts de l'action, traduction, notes et introduction de Jean-Pierre Cléro, Paris, L’unebévue éditeur.

BERGANDI, Donato (2009), «Lo sviluppo sostenibile tra utopia e realtà politica », dans Antonio FARINA, et Massimo RUSSO (dir.), I nuovi paradigmi dello sviluppo. Scienze sociali e scienze ecologiche a confronto, Trieste, GoliardicaEditrice, 9-36.

BERGANDI, Donato (2013), «Epilogue: The epistemic and practical circle in an evolutionary, ecologically sustainable society », dans Donato Bergandi (dir.), The Structural Links between Ecology, Evolution and Ethics. The Virtuous Epistemic Circle, Dordrecht, Springer, p. 151-158.

BERGANDI, Donato et Fabienne GALANGAU-QueRAT (2008), « Le développement durable : les racines environnementalistes d'un paradigme », Aster, vol. 46, p. 31-43.

BERGANDI, Donato et Patrick BLANDIN (2012), « De la protection de la nature au développement durable : genèse d'un oxymore éthique et politique », Revue d'histoire des sciences, vol. 65, n 1, p. 103-142.

BLANDIN, Patrick (2009), De la protection de la nature au pilotage de la biodiversité, Versailles, Éditions Quæ.

DAHL, Robert A. (1968), « A critique of the ruling elite model », dans Robert A. DAHL, et Deane E. NEUBAuER (dir.), Readings in Modern Political Analysis, Englewood Cliffs, New Jersey, Prentice-Hall, Inc., p. 268-276.

EISENHOwER, Dwight David (1961), Discours d'adieu prononcé par le Président Dwight David Eisenhower, traduction de Pascal Delamaire, [en ligne], [http://icp.ge.ch/po/cliotexte/deuxieme-moitiedu-xxe-siecle-guerre-froide/discours.eisenhower.html], (22 novembre 2013).

DEWEY, John (1944), « Some questions about value », The Journal of Philosophy, vol. 41, n 17, p. 449-455.

GINGRAS, Anne-Marie (2009), Médias et démocratie. Le grand malentendu, Sainte-Foy, Québec, Presse de l'Université du Québec.

HABERMAS, Jürgen, (1981), The Theory of Communicative Action, Boston, Beacon Press.

HABERMAS, Jürgen, (1996), Between Facts and Norms, Cambridge, MA, MIT Press.

KANT, Emmanuel ([1785] 1904), Fondements de la métaphysique des møurs, traduction de H. Lachelier, Paris, Librairie Hachette et $C^{\text {ie }}$.

KELLER, Suzanne (1963), Beyond the Ruling Class: Strategic Elites in Modern Society, New York, Random House.

KIMBALl, James W. (1951), « Game conservation and ecology », The Scientific Monthly, vol. 73, n 6, p. 353-355.

MARX, Karl ([1871] 1975), La Guerre civile en France-1871, Paris, Éditions sociales.

MARX, Karl et Friedrich ENGELS([1845] 1968), L'idéologie allemande, Paris, Éditions sociales.

MILL, John Stuart ([1861] 1877), Le gouvernement représentatif, traduction et introduction de C.B. Dupont-White, troisième édition, Paris, Guillamin et $C^{\mathrm{ie}}$ Éditeurs.

MILL, John Stuart ([1863] 1889), L'utilitarisme, traduction de P.-L. Le Monnier, deuxième édition revue, Paris, Félix Alcan.

MOORE, George Edward (1922), Philosophical Studies, London, Routledge and Kegan Paul.

OBER, Josiah (1996), The Athenian Revolution, Princeton, New Jersey, Princeton University Press.

Éthique publique, vol. 16, $n^{\circ} 1$ | 2014 
PARETO, Vilfredo (1968), The Rise and Fall of Elites: An Application of Theoretical Sociology, introduction de H. L. Zetterberg, Totowa, New Jersey, Bedminster Press.

PINCHOT, Gifford (1910), The Fight for Conservation, New York, Doubleday, Page \& Company. PINCHOT, Gifford (1947), Breaking New Ground, New York, Harcourt, Brace and Company.

RECLUS, Élisée (1905), L'Homme et la terre, vol. I-VI, Paris, Librairie universelle.

ROTHKOPF, David (2008), Superclass : The Global Power Elite and the World They Are Making, New York, Farrar, Straus and Giroux.

STOKES, C. Susan (1998), « Pathologies of Deliberation », dans Jon Elster (dir.), Deliberative Democracy, Cambridge, Cambridge University Press, p. 123-139.

\section{Notes}

1 Sur la sphère publique, voir aussi Gingras (2009, chapitre 1).

2 Voir aussi Marx et Engels ([1845] 1968).

3 Voir aussi Mill ([1863] $1889: 14)$.

4 Le Département d'État américain a donné un avis favorable concernant les conséquences environnementales du projet sur la base d'une étude développée par une société d'études d'impact (Environment Resources Management), recommandée par TransCanada, qui compte la société pétrolière parmi l'un de ses clients majeurs. Voir http://www.foe.org/news/blog/keystone-xl-pipeline-influencescandal.

5 James W. Kimball (1912-?) était un spécialiste de la vie sauvage qui fut directeur de la Division of Game \& Fish de l'état du Minnesota.

\section{Pour citer cet article}

\section{Référence électronique}

Donato Bergandi, «Environnement, éthique et politique : les limites d'une démocratie inaboutie et leurs conséquences néfastes sur la protection de la nature », Éthique publique [En ligne], vol. 16, $\mathrm{n}^{\circ} 1$ | 2014, mis en ligne le 22 août 2014, consulté le 08 janvier 2015. URL : http:// ethiquepublique.revues.org/1364 ; DOI : 10.4000/ethiquepublique.1364

\section{À propos de l'auteur}

\section{Donato Bergandi}

Donato Bergandiest professeur au Muséum national d'Histoire naturelle de Paris. Ses activités de recherche se situent entre la philosophie de l'écologie, la philosophie de la biologie et l'éthique de l'environnement. En plus du débat transdisciplinaire holisme-réductionnisme dans les sciences de la vie, il a ouvert ses sujets d'étude aux problématiques de la conservation de la biodiversité et de l'influence de la pensée scientifique écologique et de ses modèles sur l'éthique environnementale et le développement durable.

\section{Droits d'auteur}

Tous droits réservés

\section{Résumés}

Les politiques publiques environnementales souffrent des effets néfastes d'une entente tacite entre élites politiques et élites économiques. Indépendamment des références philosophicopolitiques, une caste oligarchique politico-économique internationale gère, de manière substantiellement unitaire et tendanciellement autocratique, les affaires environnementales selon le modèle du développement durable, matérialisation d'une perspective utilitariste, anthropocentrique et ressourciste qui, essentiellement, considère que la biodiversité n'est rien 
d'autre qu'une réserve de ressources naturelles à la disposition de l'humanité. Désormais, une double transition éthique et politique est nécessaire pour préserver l'intégrité des systèmes naturels et pour soutenir le développement des sociétés humaines.

Environmental public policies are suffering the harmful effects of a tacit agreement between political and economical elites. Heedless of philosophical-political references, an international politico-economical oligarchic caste is largely united around dealing with environmental issues based on the sustainable development model, which is an expression of a utilitarian, anthropocentric perspective. Moreover, for this model biodiversity is in the main merely a reservoir of natural resources for human use. A dual transition - both ethical and political - is thus urgently needed to preserve the integrity of natural systems and support the development of truly human societies. 